\title{
Is there a volume-quality relationship within the independent treatment centre sector? A longitudinal analysis
}

Florien Margareth Kruse ${ }^{1 *}$ D, M. C. van Nieuw Amerongen², I. Borghans ${ }^{2}$ A. S. Groenewoud ${ }^{1}$, E. Adang ${ }^{3}$ and P. P. T. Jeurissen ${ }^{1,4}$

\begin{abstract}
Background: The number of independent treatment centres (ITCS) has grown substantially. However, little is known as to whether the volume-quality relationship exists within this sector and whether other possible organisational factors mediate this relationship. The aim of this study is to gain a better understanding of such possible relationships.

Methods: Data originate from the Dutch Health and Youth Care Inspectorate (IGJ) and the Dutch Patients Association. We used longitudinal data from 4 years (2014-2017) including three different quality measures: 1) composite of structural and process indicators, 2) postoperative infections, and 3) patient satisfaction. We measured volume by the number of invasive treatments. We adjusted for three important organisational characteristics: (1) size of workforce, (2) chain membership, and (3) ownership status. For statistical inference, random effects analysis was used. We also ran several robustness checks for the volume-quality relationship, including a fractional logit model.

Results: ITCs with higher volumes scored better on structure, process and outcome (i.e. postoperative infections) indicators compared to the low-volume ITCs - although only marginally on outcome. However, ITCs with higher volumes do not have higher patient satisfaction. There is a decreasing marginal effect of volume - in other words, an L-shaped curve. The effect of the intermediating structural factors on the volume-quality relationship (i.e. workforce size, chain membership and ownership status) is less clear. Our findings suggest that chain membership has a negative influence on patient satisfaction. Furthermore, for-profit providers scored better on the Net Promoter Score.

Conclusions: Our study shows with some certainty that the quality of care in low-volume ITCs is lower than in high-volume ITCs as measured by structural, process and outcome (i.e. postoperative infection) indicators. However, the size of the effect of volume on postoperative infections is small, and at higher volumes the marginal benefits (in terms of lower postoperative infections) decrease. In addition, volume is not related to patient satisfaction. Furthermore, the association between the structural intermediating factors and quality are tenuous.
\end{abstract}

Keywords: Independent treatment centres, Volume, Quality of care, Outpatient procedures

\footnotetext{
* Correspondence: florien.kruse@radboudumc.nl

${ }^{1}$ IQ healthcare, Radboud University and Medical Center, Nijmegen, The

Netherlands

Full list of author information is available at the end of the article
}

(c) The Author(s). 2019 Open Access This article is distributed under the terms of the Creative Commons Attribution 4.0 International License (http://creativecommons.org/licenses/by/4.0/), which permits unrestricted use, distribution, and reproduction in any medium, provided you give appropriate credit to the original author(s) and the source, provide a link to the Creative Commons license, and indicate if changes were made. The Creative Commons Public Domain Dedication waiver (http://creativecommons.org/publicdomain/zero/1.0/) applies to the data made available in this article, unless otherwise stated. 


\section{Background}

Independent treatment centres (ITCs) are enjoying a growing market share in low-risk invasive ambulatory treatments such as cataract surgery and carpal tunnel syndrome [1-3]. The growth in ITC market share has been made possible by advances in technology, which have enabled more invasive treatments to be relocated from inpatient hospital care to ambulatory care settings [4]. In the United States (US), between 2000 and 2010, the number of Medicare-certified independent ambulatory surgery centres (referred to as ASCs in the US) increased on average by $5.4 \%$ per year [1]. In the United Kingdom (UK), the National Health Service (NHS) has increased the number of commissioned ITCs to improve accessibility and reduce waiting lists [2]. The Netherlands experienced a growth in the number of ITCs (in terms of the number of locations at which care is provided, or 'ITC locations'), of $87 \%$ between 2009 and 2016 [5]. Although ITCs still have a small share of $3.8 \%$ of total reimbursable care in the Netherlands in 2016, for some procedures their share is considerably higher; for example, ITCs now provide $18.4 \%$ of the total ophthalmological procedures and $18.2 \%$ of the dermatological treatments [6].

The increasing importance of ITCs as providers of health care demands an understanding of the organisational factors that contribute to safe and effective care provision; however, there has been a paucity of research on this topic. Instead, most research on the ITC sector is concerned with comparing ITCs with general hospitals, and these studies often have equivocal results [710]. The volume-quality relationship is of particular interest in the ITC sector because organisational scale is one of the key factors in understanding efficiency.

\section{Dutch ITC market}

The Dutch ITC market has some distinctive characteristics. It consists of non-profit centres providing reimbursable care from the statutory benefit package as well as for-profit centres offering non-reimbursable care. In the Netherlands, providers offering reimbursable medical specialist care (e.g. carpal tunnel syndrome and phlebology) from the statutory benefit package are formally prohibited from allocating any possible profits as a compensation for equity capital. Hence, stand-alone for-profit centres are clinics providing nonreimbursable care (e.g. refraction surgery and aesthetic surgery without GP referral). Many ITCs offer reimbursable and non-reimbursable care, but since they fall under the regulatory framework of reimbursable care they are strictly speaking non-profit institutions. The umbrella term 'ITCs' used throughout this paper refers to both non-profit and forprofit centres. Furthermore, the Dutch ITC market consists of ITC locations that are affiliated to health care chains as well as ITC locations that are sole proprietorship ITCs. The
Dutch ITC market is heavily concentrated: four of the largest chains account for $32 \%$ of the total revenue [11]. Physicians working in ITCs can be working solely for an ITC but can also be partly employed by a hospital. When general physicians are working for both an ITC and a hospital, these physicians are generally on the payroll of both providers.

\section{Volume-quality relationship}

The volume-quality relationship differs by procedure, according to the level of risk associated with it and the frequency with which hospitals undertake it (i.e. volume). Luft et al. were the first to publish on the volume-quality relationship and identified the importance of the type of procedure to the relationship [12]. Subsequently, the volumequality relationship for high-risk, inpatient procedures has been well studied. (Although, the majority of the studies neglect the intermediating factors [13].) It has been found that lower volumes are associated with worse outcomes often measured in postoperative mortality [14-16].

However, the contemporary debate regarding the volume-quality relationship focuses primarily on these high-risk, low-volume, inpatient procedures [17], and both low-risk, high-volume procedures and outpatient procedures have received much less attention in recent years. Some studies have examined the volume-quality relationship in low-risk, high-volume procedures but these have focused mainly on total knee and hip arthroplasty, and hernia repair surgery $[15,16,18-22]$. Moreover, almost all studies of the volume-quality relationship analyse inpatient hospital data and do not take into account care performed in outpatient settings [15-17]. Two papers by Chukmaitov et al. [23, 24] are rare exceptions, but their contribution to the evidence on the volume-quality relationship for low-risk outpatient treatments is limited because their data originates from Florida alone and is relatively outdated at the time of writing (i.e. 1997 and 2004) [23, 24].

The volume-quality relationship can move into two directions: 1) volume drives quality, and/or 2) quality drives volume $[25,26]$. The first direction, wherein volume drives quality, is based on the hypothesis that 'practice makes perfect'. This hypothesis reasons that quality is improved by harvesting experience - a learning effect which is comprised of both individual learning (i.e. experience of the surgeons) and organisational learning (i.e. skills and experience of the team and care locations) [27]. The volume-quality relationship can also be more static, meaning that high-volume providers will provide better outcomes irrespective of the experience of the provider [28]. The alternative direction of this relationship, wherein quality drives volume, is based on the hypothesis that providers that demonstrate a good quality of care will attract more patients. It is important to note 
that the volume-quality relationship could be characterised by either a linear or a non-linear trend [29, 30].

The theoretical framework and the empirical literature are largely focused on low-volume and high-risk treatments. (High-risk in this context does not necessarily entail a high-risk of mortality or of other severe outcomes, but it denotes negative outcomes that occur frequently.) We cannot expect that this theory can be applied directly to the ITC sector because the nature of the treatments is so fundamentally different. (The procedures are low-risk so the frequency of negative outcomes is lower than in high-risk procedures.). Hence this research adopts the null-hypothesis that there is no association between volume and quality outcomes.

\section{Mediating factors}

To identify factors that might mediate the volume-quality association, we formulated three secondary hypotheses. The first hypothesis states that a larger workforce results in higher quality. This reflects organisational learning whereby a bigger team is associated with more internal learning, support and control, and that this then increases the quality of care. One earlier study highlighted the importance of capacity and staffing as a mediating factor in the volume-quality relationship [31].

The second hypothesis holds that chain membership leads to better quality of care. Chain-affiliated ITCs could in theory provide better quality of care, since these ITCs may enjoy the benefits of greater access to resources. The availability of complementary medical and technical support services could possibly foster broader organisational knowledge [32, 33].

The third hypothesis postulates that non-profit ITCs provide better quality of care than for-profit ITCs. Three possible explanations for this hypothesis are as follows. One theory holds that non-profit organisations will outperform for-profit entities when there is asymmetry of information in favour of the provider [34] because, according to this theory, for-profit organisations would be more inclined to game the system as a result of this asymmetry. A second theory postulates that non-profit organisations specifically strive to maximise quality, whereas for-profit ITCs aim to maximise profit for their investors [35, 36]. Furthermore, in the Netherlands, health insurers have the legal discretion to selectively contract health care providers [37]. Non-profit providers may be more incentivised to constantly improve their care because they have to compete in terms of price and quality to obtain these contracts while for-profit providers do not. However, other theories instead predict that for-profit entities outperform non-profit providers on measurable quality outputs because for-profit providers are more likely to focus on these transparent quality outputs and theoretically will outperform on them [35]. It is important to note that these theories originate from the hospital sector and we do not know in how far they hold for the ITC sector.

In summary, this study aims to explore the question of whether volume is associated with quality in the ITC sector and, in addition, identify possible mediating structural factors (i.e. workforce size, chain membership and ownership status).

\section{Methods}

Data

Our data originated from the Dutch Health and Youth Care Inspectorate (IGJ). IGJ uses a framework of risk indicators for the supervision of ITCs [38]. Since 2008, IGJ has been collecting annual information by means of a mandatory quality assessment questionnaire, completed by the ITC locations themselves (Additional file 1). We constructed a dataset ranging from the years 2014 to 2017. IGJ inspectors were involved in deciding which indicators were most suitable for this study. The inclusion criteria for ITCs were that they should provide invasive treatments and offer at least one of the following specialties: ophthalmology, dermatology, orthopaedics or aesthetic surgery. The dataset included 338 ITCs and 206 of these had at least 3 years of observations.

Patient satisfaction data was obtained from the Dutch Patients Association (Patientenfederatie), which collects information through a patient rating website (ZorgkaartNederland.nl). This platform is a well-known website, with around 700,000 ratings where patients, if they wish, can leave their feedback. The scores are on a 0 to 10 scale and are based on the ratings per ITC location regarding treatment, information provision, listening competency, handling by staff, accommodation, and experience in scheduling an appointment. Patient ratings between 2014 and 2017 were included. Of those ITCs included in the IGJ dataset, 166 ITCs had patient ratings. We followed the methodology of Kool et al. [39] to further restrict these scores to providers with 30 or more patient ratings, leaving 80 ITCs with a total of 19,294 ratings.

A description of how the data was merged between the patient ratings and the IGJ data can be found in Additional file 2.

\section{Variables}

Volume was measured by the number of invasive treatments. We also constructed a percentile-based categorisation of the annual number of invasive treatments in order to gain a better understanding of how low-volume ITCs (up to \pm 300 ), lower-medium-volume ITCs (up to \pm 890 ), higher-medium-volume ITCs (up to \pm 2130 ) and high-volume ITCs perform relative to each other. (We follow the advice of Luft et al. to compare various indicators of volume [40].) Workforce size is indicated by 
the full-time equivalent (FTE) of physicians and nurses. Dichotomous variables were made for chain affiliation (i.e. single location versus multiple locations) and for ownership status (i.e. non-profit versus for-profit).

We used three alternative measures for different dimensions of quality: (i) a composite of structural and process indicators; (ii) postoperative infections; (iii) patient ratings. The structural and process indicators were based upon the Donabedian model [41] and are dichotomous variables with values representing ' 1 ' as good performance and ' 0 ' as poor performance. We constructed a composite of structural and process indicators based upon the annual sum of the Z-scores of the seven categorical structural and process quality indicators (Table 1). Z-scores were used to assign weights to the different quality measures. The data from 2017 did not have the seven categorical quality indicators, hence no observations for that year could be used for the composite measure score.

Medical quality was assessed by the rate of postoperative infections: the lower the rate the better the medical quality. This measure has been used for this purpose in other studies [42-44]. With the patient satisfaction data, five indicators were created: (1) promoter (average score of 9 or higher); (2) detractor (average score of 6 or lower); (3) Net Promoter Score (NPS) (i.e. the percentage of promoters minus the percentage of detractors per provider) [45]; (4) average score above 7; and (5) average score above 8 . The last two measures are not based on the NPS classification but are defined to identify other possible cut-off points. Patient ratings are not normally distributed because patients who are satisfied or dissatisfied generally rate their providers more frequently than people with neutral opinions; the indicators above address this complication.

To adjust for possible confounders, four types of control variables were included in the models. Firstly, ASA physical status classification II and ASA III [46] were used to adjust for case-mix differences since this could possibly affect quality. Secondly, we adjusted for the different medical specialities since the different specialities

Table 1 The seven structural and process quality indicators

Whether an independent treatment centre...
is reachable 24/7
has a system whereby the performance of their personnel is reviewed
has an arrangement in place for dysfunctional personnel
uses a questionnaire that inquires patient reported experiences or
outcomes
classified the American Society of Anesthesiologists (ASA) physical
status (i.e. severity) of their patients
screened for delirium
has a collaboration with (a) hospital(s)

have different quality risks. Lastly, the models account for year-dependent effects.

One of the assumptions is that locations within the same chain behave similarly. To account for chain clustering, we created unique chain identifiers.

\section{Data analysis \\ Descriptive statistics}

Because this study uses panel data, the overall mean, the within-provider variances and between-providers variances were calculated. The differences between the overall and between variances is that the between variances use the mean of the panel data while the overall mean calculates the weighted mean of the panel data, whereby the weights are given by the number of observations in the panel data.

\section{Linearity of the volume-quality relationship}

For the volume-quality relationship in ITCs, linearity of the curve is tested by re-expressing the number of invasive treatments. The number of invasive treatments is right-skewed and therefore transformed down the ladder of powers - to a squared root (SQRT), a cube root (Ushaped curve) and logarithmic function (L-shaped curve) [47]. The fit of the re-expressed values is based on the Akaike information criterion (AIC) [48]. The lower the AIC score, the better the model resembles the data. To further explore this assumption, we will also report the augmented component plus residual plots according to the method proposed by Mallows [49] (consult Additional file 2 for a longer description).

\section{Explanatory regressions}

We used a Random Effects (RE) model which clusters the observations within the unique provider and/or chain identifiers. (The Hausman's test preferred the RE model over the Fixed Effects estimates [50].) The continuous dependent variables (i.e. composite structural and process indicators, postoperative infections and NPS) are estimated with a linear RE model. For postoperative infections, the linear RE models only included those providers that had above 0 postoperative infections and with at least 50 invasive treatments to prevent outliers. For the binary dependent variables (i.e. promoter, detractor, average score above 7 , average score above 8 ) a RE logistic model was used. In addition, we performed an analysis pooling all postoperative infections and invasive treatments over the 3 years to overcome the exclusion of the smaller providers with less than 50 invasive treatments and possibly include ITCs that had 0 postoperative infections in 1 year, but during the course of 3 years, are more likely to have above 0 postoperative infections. Providers with observations for only 1 or 2 years were excluded from this analysis. When providers 
had 4 years of observations, we took the average of the 4 years to subtract one average year from the total 4 years of observations to get 3 years of pooled observations.

The correlation between workforce size and volume can substantially distort the analysis therefore all the models were tested for multicollinearity with the variance inflation factor (VIF). We find that none of the VIF values were greater than 10 which, as a rule of thumb, suggests the models are not affected by multicollinearity [51] (Additional file 3).

For all the RE models, we tested whether observations were clustered within ITC locations and chain membership using the likelihood-ratio test. For models using longitudinal data, the test identified clustering within ITC locations. For the pooled 3-year data and the patient ratings models, the test identified clustering within chains.

\section{Robustness checks}

We performed a fractional logit model for postoperative infections in order to include the zeros and accommodate the proportional distribution, which the RE model is unable to do. The exclusion of the zeros could potentially penalise low-volume ITCs since they are more likely to have zero postoperative infections. The postoperative infections are included in the fractional logit model as values between 0 and 1 . The fractional logit regression model can account for intragroup correlations in the panel dataset, however it is less capable than the $\mathrm{RE}$ model of accommodating complexities such as the unbalanced panel structure.

A second robustness check addresses the problem that within the dataset it is not possible to directly link specific treatments with specific postoperative infections because when ITCs have multiple specialties $(43 \%$ of providers) total volume is assessed. To correct for this, the models with postoperative infections were also specifically run including only aesthetic surgery and postoperative infections after aesthetic surgery.

Furthermore, as a low number of invasive treatments can potentially skew the percentage of postoperative infections, an additional robustness check was performed whereby the cut-off point was set at 100 invasive treatments instead of 50 . In addition, we ran the results without including the case-mix factors since many ITCs had missing values for the case-mix factors, which means the models lost a high number of providers by including case-mix as a control.

\section{Results}

\section{Descriptive statistics} ITC characteristics

The number of invasive treatments shows substantial variation between ITCs with, on average, 1572 invasive treatments per ITC but a high standard deviation of
1882 (Table 2). With a median of 886 invasive treatments (not shown in the Table 2), this data is rightskewed. The average FTE of physicians is 2.3 physicians, with a standard deviation of 2.5 which is relatively high. Compared to the FTE of physicians, the average FTE of nurses is lower, at 1.5 nurses, with a standard deviation of 3.6, which, as for physicians, is high. Most providers are non-profit centres: $32 \%$ of the locations are forprofit. Additional file 4 summarises the differences between non-profit ITCs and for-profit ITCs with respect to volume and chain-affiliation. In brief, the non-profit ITCs are bigger than the for-profit ITCs: non-profit ITCs completed a higher number of invasive treatments. Non-profit ITCs are also more often chain-affiliated, and non-profit chains have more ITC locations than the forprofit chains. In addition, sole-proprietorship ITCs perform a lower number of invasive treatments than the chain-affiliated ITCs, and this is the case for both forprofit ITCs and non-profit ITCs.

\section{(Composite) structural and process indicator(s)}

Most of the ITCs - around $70 \%$ to $80 \%$ - comply with four of the individual structural and process quality indicators, indicating that most centres perform well on these measures (Table 2). Three indicators present much lower scores of around 30 to $60 \%$. Firstly, $36 \%$ of ITCs have no collaboration agreement with any hospital in case of emergency. Secondly, $52 \%$ of the ITCs did not use an ASA classification. And, thirdly, 66\% did not screen for delirium. All of these are obligatory for ITCs conducting invasive treatments. The within standard deviation of the structural and process indicators illustrates that these indicators change over the years within ITCs. This is partly due to the fact that the weights per year could deviate. The mean of the structural and process composite is almost zero, which is as expected since the composite is based upon Z-scores. The standard deviation is 3.7, which is relatively high and demonstrates that there is substantial variance between ITCs. In order to get a sense of the scale of this composite, it ranges from -13.1 to 5.8. (We would like to stress that this variation of Z-scores is based upon the sum of Z-scores of the seven structural and process indicators. The individual Z-scores show much less variation.)

\section{Outcome indicators and patient satisfaction}

The percentage of postoperative infections is low with approximately 3 in 1000 invasive treatments resulting in postoperative infections (Table 2). For those providers with at least one patient with a postoperative infection and which performed 50 or more invasive treatments, the rate was slightly higher, at 5 in 1000 invasive treatments resulting in a postoperative infection. For the outcomes related to the patient satisfaction ratings, the mean score 
Table 2 Summary statistics 2014-2017

\begin{tabular}{|c|c|c|c|c|}
\hline & Overall mean \pm SD & Between SD & Within SD & $N(n)$ \\
\hline \multicolumn{5}{|l|}{ Characteristics ITCS } \\
\hline Number of invasive treatments & $1571.85 \pm 1881.56$ & 1693.96 & 819.81 & $941(338)$ \\
\hline FTE physicians & $2.32 \pm 2.45$ & 2.35 & 1.04 & 941 (338) \\
\hline FTE nurses & $1.49 \pm 3.55$ & 3.34 & 1.14 & 941 (338) \\
\hline Number of locations & $2.61 \pm 3.11$ & 2.73 & 0.82 & 941 (338) \\
\hline Chain membership & $0.40 \pm 0.49$ & 0.47 & 0.16 & $941(338)$ \\
\hline Non-profit providers & $0.68 \pm 0.47$ & 0.47 & 0.00 & $941(338)$ \\
\hline \multicolumn{5}{|l|}{ Composite Quality indicators } \\
\hline Reachable 24/7 & $0.67 \pm 0.47$ & 0.31 & 0.40 & $716(313)$ \\
\hline Personnel functioning system & $0.78 \pm 0.41$ & 0.38 & 0.21 & $716(313)$ \\
\hline Personnel malfunctioning system & $0.78 \pm 0.41$ & 0.36 & 0.22 & $716(313)$ \\
\hline Patient satisfactory questionnaire & $0.88 \pm 0.33$ & 0.32 & 0.16 & $716(313)$ \\
\hline ASA classification known & $0.48 \pm 0.50$ & 0.46 & 0.21 & $716(313)$ \\
\hline Screening delirium & $0.34 \pm 0.48$ & 0.42 & 0.23 & $716(313)$ \\
\hline Collaboration with (a) hospital(s) & $0.64 \pm 0.48$ & 0.45 & 0.20 & $716(313)$ \\
\hline Structural and process composite & $-0.00 \pm 3.31$ & 3.19 & 1.48 & $716(313)$ \\
\hline \multicolumn{5}{|l|}{ Quality outcomes } \\
\hline Percentage infections & $0.28 \pm 1.14$ & 0.96 & 0.79 & 877 (318) \\
\hline Percentage infections ( $>0$ postoperative infections $\&>=50$ invasive treatments) & $0.47 \pm 0.62$ & 0.65 & 0.29 & $412(189)$ \\
\hline Average patient satisfaction score & $8.74 \pm 1.17$ & 0.40 & 1.12 & $19,338(80)$ \\
\hline Ratio promoters over total number of observations per provider & $0.52 \pm 0.50$ & 0.17 & 0.47 & $19,338(80)$ \\
\hline Ratio detractors over total number of observations per provider & $0.03 \pm 0.17$ & 0.04 & 0.17 & $19,338(80)$ \\
\hline Ratio 7 or more over total number of observations per provider & $0.95 \pm 0.22$ & 0.04 & 0.21 & $19,338(80)$ \\
\hline Ratio 8 or more over total number of observations per provider & $0.84 \pm 0.36$ & 0.09 & 0.35 & $19,338(80)$ \\
\hline Net Promoter Score (in ratio) & $0.55 \pm 0.19$ & 0.19 & 0.06 & $118(55)$ \\
\hline \multicolumn{5}{|l|}{ Control variables } \\
\hline Specialism ophthalmology & $0.23 \pm 0.42$ & 0.39 & 0.07 & $941(338)$ \\
\hline Specialism dermatology & $0.37 \pm 0.48$ & 0.47 & 0.13 & $941(338)$ \\
\hline Specialism orthopaedics & $0.11 \pm 0.31$ & 0.31 & 0.05 & $941(338)$ \\
\hline Specialism aesthetic surgery & $0.59 \pm 0.49$ & 0.47 & 0.18 & $941(338)$ \\
\hline Ratio ASA II over total number of patients & $0.13 \pm 0.16$ & 0.14 & 0.08 & $622(241)$ \\
\hline Ratio ASA III over total number of patients & $0.01 \pm 0.06$ & 0.04 & 0.04 & $623(242)$ \\
\hline \multicolumn{5}{|l|}{ Robustness check } \\
\hline Number of aesthetic invasive treatments & $502.40 \pm 1269.82$ & 972.17 & 844.36 & $488(211)$ \\
\hline Percentage infections after aesthetic surgery & $0.90 \pm 6.81$ & 7.76 & 4.16 & $449(182)$ \\
\hline
\end{tabular}

is 8.7 with an overall standard deviation of 1.2. The mean rate of promoters lies around $52 \%$ per provider, while the mean rate of detractors accounts for $3 \%$. The score of 7 or higher was given by $95 \%$ of the patients, and $84 \%$ score 8 or higher. The NPS accounts for $55 \%$.

\section{Control variables}

There is some diversity in which specialties are offered by ITCs (Table 2). Most of the ITCs offer aesthetic surgery (59\%), whereas there are fewer orthopaedic ITCs (11\%). The summary statistics further show that on average $13 \%$ of the ITC patients have mild systemic diseases, ASA II, and only $1 \%$ are patients with severe systemic diseases, ASA III.

\section{Explanatory statistics \\ Linearity}

The AIC scores of the different models are exhibited in Table 3. The relationship is non-linear for all the quality indicators; the AIC rates the logarithmic curve as the best fit for all the quality indicators. 
To visualise this relationship, Fig. 1 shows the augmented partial residuals on the $y$-axis and on the $x$-axis the total number of invasive treatments. The grey line depicts the linear trend and the green line fits to the potential non-linear curve. Unlike the AIC scores, both lines in Fig. 1 show that there is no clear non-linear trend regarding the association between volume and the structural and process indicators. Likewise, the visualisation does not present a non-linear curve for the relationship between the NPS and volume. In contrast, the observations with postoperative infections delineate a distinctive negative logarithmic function, similar to the trend found within the pooled 3-year data. For postoperative infections, the inflection point seems to occur at roughly 2000 invasive treatments; thereafter the impact of size seems to diminish.

\section{Volume-quality relationship}

The logarithmic curve shows a positive relationship between the composite of the structural and process indicators and volume (Table 4, model I). For ITCs with postoperative infections and with 50 or more invasive treatments, the percentage of postoperative infections declines with the number of invasive treatments (Table 4, model II). In other words, a $10 \%$ increase in the number of invasive treatments is associated with a reduction in the annual number of postoperative infections by 0.03 percentage points $\left(-0.339^{*} \log (1.10)\right)$. When the 3 years of observations are pooled together, the relationship persists but the effect size weakens to a 0.009 percentage point reduction in postoperative infections (0.094* $\log (1.10)$ (Table 4, model III). This may indicate that higher denominators and/or the exclusion of providers in the annual models $(<50$ invasive treatments or 0 postoperative infections) reduce the effect size. Table 4 , Model IV, suggests that low-volume ITCs have a higher chance of postoperative infections than high-volume ITCs. Patient satisfaction has a weak association with the number of invasive treatments. The mean patient rating declines with a higher number of invasive treatments (Table 5). In addition, the chance of having promoters and ratings above 8 declines with volume. All three are only statistically significant on a $90 \%$ confidence level. In contrast, the NPS, ratings above 7 and the number of detractors do not display a relationship with the number of invasive treatments.

\section{Mediating structural factors}

The FTE of physicians and nurses seems to be unrelated to the structural and process quality indicators (Table 4, model I). There is no evidence of a relationship between the FTE professionals and the rate of postoperative infections in either the annual or the pooled data (Table 4, model II \& III). Finally, patient satisfaction is also not significantly related to the FTE of physicians and nurses (Table 5).

Structural and process quality indicators suggest that chain membership has no effect on performance (Table 4, Model I). The positive relationship between chain membership and postoperative infections indicates that there are, on average, higher rates of postoperative infections in chain-affiliated ITCs (Table 4, model II). However, the confidence interval is only $90 \%$ and the relationship dissolves when the data is pooled (Table 4, model III). Patient satisfaction data illustrate a negative and consistent relationship with chain membership, but only on a $90 \%$ confidence interval (Table 5). The only patient satisfaction indicator which shows chain membership having no effect is the number of patients given ratings of 9 or above (i.e. promoters).

No association was found between ownership and the structural and process indicators (Table 4, model I). For the annual data analysis, non-profit providers do not seem to have a significantly higher or lower percentage of postoperative infections than for-profit providers (Table 4, model II). However, when the data is pooled, the non-profit providers are associated with higher percentages of postoperative infections (Table 4, model III). It is likely therefore that the relationship between ownership and postoperative infections can only be detected with the inclusion of higher denominators or the possible inclusion of centres that could not be included in the annual data analysis (i.e. those with $<50$ invasive treatment and zero postoperative infections). Regarding the patient ratings, only the NPS is significantly lower for non-profit providers compared to forprofit providers (Table 5).

Table 3 AIC scores

\begin{tabular}{lllll}
\hline & Linear & SQRT & Cubic & Logarithmic \\
\hline Structural and process composite & 2152 & 2147 & 2144 & 2138 \\
Postoperative infections & 377.4 & 352.0 & 339.9 & 311.7 \\
Pooled data - postoperative infections & 40.90 & 39.10 & 38.60 & 38.30 \\
Aesthetic postoperative infections & 408.6 & 403.7 & 400.5 & 393.5 \\
Patients ratings - mean score & 59,435 & 59,433 & 59,432 & -128.3 \\
NPS & -128.1 & -128.2 & 59,430 & -128.5 \\
\hline
\end{tabular}



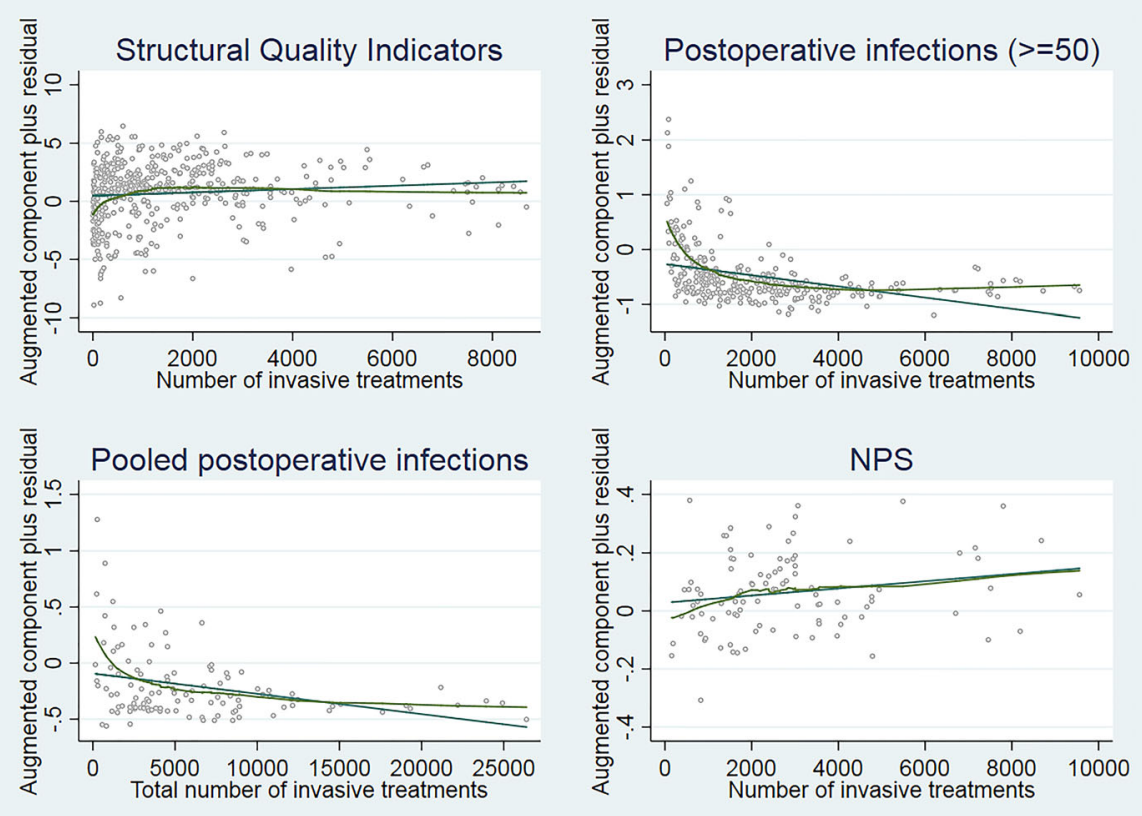

Fig. 1 Visualisation non-linear volume-quality relationship

Table 4 Relationship between the composite structural and process quality indicators or postoperative infections and ITC characteristics

\begin{tabular}{|c|c|c|c|c|}
\hline & Model I & Model II & Model III & Model IV \\
\hline Type of outcome variable & $\begin{array}{l}\text { Composite structural and process } \\
\text { quality indicator }\end{array}$ & $\begin{array}{l}\text { Percentage } \\
\text { postoperative infections }\end{array}$ & $\begin{array}{l}\text { Percentage } \\
\text { postoperative infections }\end{array}$ & $\begin{array}{l}\text { Percentage } \\
\text { postoperative infections }\end{array}$ \\
\hline Type of model used & RE-Linear & RE-Linear & RE-Linear & RE-Linear \\
\hline Type of data used & Annual data & Annual data & Total over 3 years & Annual data \\
\hline Log invasive treatments & $0.418^{* * *}(0.089)$ & $-0.339^{* * *}(0.033)$ & $-0.094^{* * *}(0.031)$ & \\
\hline $\begin{array}{l}\text { Highest quantile invasive } \\
\text { treatments }\end{array}$ & & & & Reference \\
\hline $\begin{array}{l}\text { Higher medium quantile } \\
\text { invasive treatments }\end{array}$ & & & & $0.029(0.096)$ \\
\hline $\begin{array}{l}\text { Lower medium quantile } \\
\text { invasive treatments }\end{array}$ & & & & $0.169(0.107)$ \\
\hline $\begin{array}{l}\text { Lowest quantile invasive } \\
\text { treatments }\end{array}$ & & & & $0.293^{* * *}(0.114)$ \\
\hline FTE number of professionals & $0.009(0.034)$ & $0.010(0.006)$ & $0.011 *(0.006)$ & $0.009(0.009)$ \\
\hline No chain membership & Reference & Reference & Reference & Reference \\
\hline Chain membership & $-0.393(0.302)$ & $0.116^{*}(0.063)$ & $-0.130(0.090)$ & $-0.100(0.074)$ \\
\hline For-profit & Reference & Reference & Reference & Reference \\
\hline Non-profit & $0.449(0.363)$ & $0.028(0.073)$ & $0.174^{* *}(0.075)$ & $0.187^{* *}(0.087)$ \\
\hline Cluster/Identifier & ID ITC & ID ITC & ID Chain & ID ITC \\
\hline Observations & 459 & 292 & 112 & 596 \\
\hline Number of groups & 211 & 145 & 72 & 236 \\
\hline
\end{tabular}

Corrected for type of specialism, case-mix (i.e. ASA II \& III) and year effects (except for the pooled data)

${ }^{* * *} p<0.01,{ }^{* *} p<0.05,{ }^{*} p<0.1$ 
Table 5 Relationship between patient ratings and ITC characteristics

\begin{tabular}{|c|c|c|c|c|c|c|}
\hline Type of outcome variable & Mean score & Promoter $(>=9)$ & Ratings $>=7$ & Ratings $>=8$ & Detractor $(<=6)$ & NPS \\
\hline Type of model & RE-Linear & RE-Logit & RE-Logit & RE-Logit & RE-Logit & RE-Linear \\
\hline Log invasive treatments & $-0.073^{*}(0.035)$ & $-0.123^{*}(0.071)$ & $-0.103(0.121)$ & $-0.174^{*}(0.099)$ & $0.182(0.151)$ & $0.027(0.025)$ \\
\hline FTE number of professionals & $0.002(0.003)$ & $0.007(0.006)$ & $0.008(0.012)$ & $0.014(0.009)$ & $-0.013(0.016)$ & $-0.00(0.002)$ \\
\hline No chain membership & Reference & Reference & Reference & Reference & Reference & Reference \\
\hline Chain membership & $-0.163^{*}(0.089)$ & $-0.133(0.184)$ & $-0.409 *(0.238)$ & $-0.390 *(0.212)$ & $0.504^{*}(0.293)$ & $-0.077^{*}(0.047)$ \\
\hline For-profit & Reference & Reference & Reference & Reference & Reference & Reference \\
\hline Non-profit & $-0.133(0.114)$ & $-0.365(0.233)$ & $-0.355(0.361)$ & $-0.446(0.288)$ & $0.037(0.429)$ & $-0.178^{* * *}(0.061)$ \\
\hline Level of measurement & Patient level & Patient level & Patient level & Patient level & Patient level & Provider level \\
\hline Cluster & ID ITC + ID Chain & ID ITC + ID Chain & ID ITC + ID Chain & ID ITC + ID Chain & ID ITC + ID Chain & ID Chain \\
\hline Observations & 16,507 & 16,507 & 16,507 & 16,507 & 16,507 & 97 \\
\hline Number of groups & 68 & 68 & 68 & 68 & 68 & 46 \\
\hline
\end{tabular}

Corrected for case-mix (ASA II \& III), type of treatment and year

${ }^{* * *} p<0.01,<0.05,{ }^{*} p<0.1$

\section{Robustness checks}

Table 6 shows the robustness checks with fractional logit regressions and the restricted model with aesthetic invasive treatments and the percentage of postoperative infections after aesthetic surgery. The fractional logit regression results support the volume-quality findings from the RE models (Table 6, Model I \& II). The only stark difference is that the lower-medium-volume ITCs also seem to perform significantly worse than the high-volume ITCs in the fractional logit regression model (Table 6, Model II). This finding possibly suggests that the inclusion of centres with zero-infections is advantageous for the relative performance of high-volume ITCs compared to the low-volume ITCs and lower-medium-volume ITCs. The restricted model with the aesthetic invasive treatments also supports the findings on the volume-quality relationship (Table 6, Model III). Lastly, the results without including the case-mix factors supports our findings in Tables 4 and 5 (Additional file 5). Interestingly, while in Table 5 there is a weak relationship between volume and patient satisfaction, without case-mix correction, all patient satisfaction indicators are negatively and significantly related to volume

Table 6 Robustness check with fractional logit models and aesthetic invasive treatments

\begin{tabular}{llll}
\hline & Model I & Model II & Model III \\
\hline Type of outcome variable & $\begin{array}{l}\text { Proportional postoperative } \\
\text { infections }\end{array}$ & $\begin{array}{l}\text { Proportional postoperative } \\
\text { infections }\end{array}$ & $\begin{array}{l}\text { Percentage postoperative infections - } \\
\text { aesthetic surgery }\end{array}$ \\
$\begin{array}{l}\text { Type of model used } \\
\text { Log invasive treatments }\end{array}$ & Annual data & Fractional logit & RE-Linear \\
Highest quantile invasive treatments & $-0.226^{* *}(0.111)$ & Annual data & Annual data \\
Higher medium quantile invasive & & Reference & $-0.566^{* * *}(0.135)$ \\
treatments & & $0.279(0.193)$ & \\
Lower medium quantile invasive & & $0.869^{* * *}(0.236)$ & \\
treatments & & $1.321^{* * *}(0.452)$ & \\
Lowest quantile invasive treatments & & $0.056^{* * *}(0.021)$ & \\
FTE number of professionals & $0.029(0.021)$ & Reference & $0.012(0.033)$ \\
No chain membership & Reference & $-0.550^{*}(0.318)$ & Reference \\
Chain membership & $-0.188(0.215)$ & Reference & $0.755^{* *}(0.317)$ \\
For-profit & Reference & $0.782^{* *}(0.339)$ & Reference \\
Non-profit & $0.558^{*}(0.310)$ & ID ITC & $-0.226(0.319)$ \\
Cluster/ldentifier & ID ITC & 596 & ID ITC \\
Observations & 555 & 113 \\
\hline
\end{tabular}


except for the NPS. Case-mix could partly mediate the volume-patient-satisfaction relationship, but this discrepancy can also be because the models had more statistical power due to the higher number of ITC locations included in the analysis. The model that restricted the analysis to ITCs with 100 or more invasive treatments (instead of 50 or more invasive treatments) gives similar results to the volume-quality relationship reported in Table 4 (also included in Additional file 5).

\section{Discussion}

The results of this study indicate that volume is associated with better performance on the structural and process indicators and on the number of postoperative infections - our outcome indicator. However, because the number of postoperative infections is generally low in low-risk surgical procedures, any increase in volume is associated with only a small decrease in the number of postoperative infections. Furthermore, our study suggests that there is a non-linear relationship between volume and quality, particularly for postoperative infections. This finding is in line with the findings from the hospital sector $[12,30]$, but contrary to the study on elective surgical procedures [18]. We find an L-shaped curve with around 2000 invasive treatments as a rough inflection point. A relationship between higher volumes and higher quality of care was also reported by Chukmaitov et al. [23], who specifically studied the ITC sector in the US and found a weak association between volume and the number of 30-day unplanned hospitalisations. The volume-quality association was also confirmed by studies scrutinising high-volume and low-risk procedures [19-22, $24,52]$, and by reviews including high-risk procedures [1416]. However, one study from the UK that looked at three elective surgical procedures (hernia repair, hip replacement and knee replacement) found no association, or of no clinical significance, between volume and quality [18].

Our models also indicate a negative relationship between volume and patient satisfaction, although with less certainty. This outcome contradicts the findings of a previous study which suggested that patients with total hip replacement surgery performed at low-volume hospitals were less satisfied than those treated in high-volume hospitals [21].

Regardless of the apparent relationship between volume and quality in this study, these findings do not provide enough evidence to reject fully the null-hypothesis because the effect size between volume and quality is small and because of limitations detailed in the limitations section. Further research should be undertaken to scrutinise the volume-quality relationship for outpatient care.

None of the three hypotheses concerning the structural mediating factors that could potentially mediate the relationship between volume and quality were supported by our study. Firstly, workforce size has no significant relationship with quality of care, and therefore our hypothesis that a bigger workforce improves quality does not hold. This outcome is contrary to one study that found a positive relationship between workforce size and quality by outpatient clinics [53]. Various studies have assessed more specifically whether surgeon volume has an effect on patient outcome. One review found a positive relationship between surgeon volume and quality of care [17]. Secondly, our study provides no evidence of a robust relationship between chain membership and quality (i.e. structure and outcome), although we did find a negative, but statistically weak (90\%), association with patient satisfaction. This goes against our second hypothesis but partly reflects the evidence that shows that concentration and multihospital systems in the US hospital sector do not lead to better quality [54-57]. Thirdly, and contrary to our third hypothesis, we did not find that non-profit providers outperform for-profit providers regarding quality of care. The international empirical evidence for the relationship between ownership and quality presents mixed results which seem to depend heavily on the context (e.g. financial incentives) [58-60]. However, our findings do indicate that for-profit providers score better on the NPS - a more businessoriented, measurable outcome - which supports the theory that for-profit providers score better on the measurable and transparent outcomes.

Our findings suggest that, given the variation in quality of care among ITCs is substantial (i.e. structural and process indicators and the postoperative infections), there are various ways of improving the efficient allocation of care. On the other hand, the descriptive statistics demonstrate that on average ITCs perform well on quality. Most ITCs comply with the structural and process quality indicators; the average chance of postoperative infections is relatively low; and the average NPS is 55\%, which is high compared to the median NPS of $16 \%$ for more than 400 companies in 28 industries [45].

To the best of our knowledge, this is the first scientific study on the quality of care in the ITC sector in the Netherlands and one of the first studies on the volumequality relationship for high-volume and low-risk procedures taking the entire ITC sector into account. These findings may help various stakeholders to understand the ITC sector better. For example, the Dutch health care inspectorate inspects the ITC sector by means of inspection interventions, which in part are guided by various indicators. Some of these indicators were part of this study. The inspectorate could further investigate the difference between low- and high-volume ITCs, preferably taking into account non-linearity when using this indicator.

These results may have important implications for patients as well. In a regulated competitive health care system, patients are empowered to choose their own health care provider and our findings illustrate that patients 
should be aware of the variation in performance within the ITC sector.

A data-related practical implication is that the available quality indicators are sub-optimal and therefore we make an appeal to stakeholders in charge to continue their commitment to enhance quality measures within the ITC sector (e.g. patient-reported outcome measures (PROMs)) and improve the quality reporting system.

\section{Limitations}

Despite the richness of our database there may be some biases. Firstly, we did not attempt to disentangle the direction of the volume-quality relationship. Likewise, the data did not allow us to study the learning curve of individual surgeons, with which we could have further explored the volume-quality relationship. We also did not have the opportunity to explore other mediating factors - for instance, the possible impact of quality improvement programmes [61].

Secondly, ITCs filled out the data questionnaire themselves and this could result in misreporting. It could, for example, lead to underreporting of postoperative infections due to a suboptimal postoperative surveillance system or it could incentivise desirable answers [62-64]. However, for these clinics there are no financial consequences based on what they have reported, so perverse incentives are minimised. For this reason, we expect the bias from self-reporting to move in the same direction (i.e. underreporting) for all ITCs.

Thirdly, patient ratings have their weaknesses, in particular potential selection bias [65]. A number of ITCs did not receive online patient rating scores therefore we performed a significance test with the total number ITCs included in our dataset and the ITCs with at least 30 patient ratings. The test found significant differences in relation to the size of the organisations (Additional file 6). This selection could potentially lead to a Type II error. Furthermore, the online patient rating scores might be subject to selection bias because the patients have to go proactively to the online patient rating website to provide their feedback; they do not receive a reminder after their treatment. We assume all providers are subject to the same bias.

Fourthly, it remains a challenging endeavour to assess the relationship between volume and postoperative infections because (i) the chance of having postoperative infections naturally increases with volume; (ii) small denominators can generate outliers; and (iii) the chance of having postoperative infections is rather low for ITC services. We have addressed this complexity by running a number of models: first, excluding the providers without postoperative infections and setting a minimum volume cut-off point; second, pooling 3 years of observations; and third, a fractional logit model as a robustness check.
Fifthly, although we obtained patient-level data for the patient ratings for this study, the other variables are at the ITC location level. In order to derive more conclusive results, patient-level data for all variables would be preferable, but this data does not (yet) exist for the entire ITC sector.

Lastly, we could not differentiate for hybrid locations - those ITCs that offer a combination of reimbursable and non-reimbursable care. Non-profit ITCs might avoid the for-profit ban with creative accounting [66].

\section{Conclusions}

Our results indicate that, in general, low-volume ITCs are more likely to provide lower quality of care for low-risk invasive ambulatory care than high-volume ITCs. ITCs with more invasive treatments score better on structure, process and outcome (i.e. fewer postoperative infections). However, the relationship between volume and postoperative infections is small and is a non-linear relationship - an L-shaped curve - which suggests a ceiling whereat the marginal benefit of higher volume ITCs diminishes. The visual representation seems to suggest that the inflection point for the rate of postoperative infections is at around 2000 invasive treatments per ITC location. In addition, higher volume does not necessarily lead to higher patient ratings, and possibly even influences patient satisfaction negatively.

The mediating factors have a more tenuous relationship with quality. The size of the workforce is not related to the three quality measures. Furthermore, our results suggest that chain membership does not improve quality of care. Instead, a negative relationship between chain membership and patient ratings seems apparent. Likewise, the theory that non-profit providers outperform for-profit providers was not supported by our findings; the relationship is equivocal. Ownership type is not related to the structural and process indicators, but the findings for the pooled postoperative infections and the NPS suggest that for-profit providers might outperform non-profit providers on those quality indicators.

\section{Additional files}

Additional file 1 Questions used from the IGJ dataset.

Additional file $\mathbf{2}$ Additional methods description.

Additional file $\mathbf{3} \mathrm{VIF}$ scores.

Additional file $\mathbf{4}$ Summary statistics divided by the type of provider and chain-membership.

Additional file 5 Robustness checks: without ASA class included as control plus results with a cut-off point of 100 invasive treatments for postoperative infections.

Additional file $\mathbf{6}$ Statistical difference between providers with and without patients' ratings. 


\section{Abbreviations}

AIC: Akaike Information Criterion; FTE: Full time equivalent; IGJ: Dutch Health and Youth Care Inspectorate; ITC: Independent Treatment Centre; NPS: Net Promoter Score; PROMs: Patient-reported outcome measures; RE: Random Effects; SQRT: Squared root; UK: United Kingdom; US: United States

\section{Acknowledgements}

We thank the Dutch Patient Association (Patientenfederatie) for providing the patient rating data from ZorgkaartNederland for this study. We thank the anonymous reviewers for their constructive comments that greatly improved this manuscript. We thank Niek Stadhouders, Inger Abma \& Nicholas Crawford for their useful input and help. Our gratitude also goes out to the inspectors who advised us on the use and interpretation of the indicators.

\section{Authors' contributions}

FK, MvNA, AG, IB, EA were responsible for the study design. FK was responsible for the data analysis and writing. MvNA provided the data and assisted with the data interpretation. EA, AG and PJ supervised the study. PJ initiated the study. MvNA, IB, AG, EA and PJ contributed to the drafting and revising of the manuscript. All authors read the manuscript and approved.

\section{Funding}

The research presented did not receive specific funding from any funding agency.

\section{Availability of data and materials}

The longitudinal IGJ datasets (2014-2017) used and analysed during the current study are available from the corresponding author on reasonable request. The data from the Dutch Patients Association that support the findings of this study are available from the Dutch Patients Association (Patientenfederatie), but restrictions apply to the availability of these data, which were used under license for the current study, and so are not publicly available. Data are however available from the authors upon reasonable request and with permission of the Dutch Patients Association.

\section{Ethics approval and consent to participate}

The research ethics committee of the Radboud University Nijmegen Medical Centre under Filenumber CMO:2019-5159 exempted this research for the Medical Research Involving Human Subjects Act (WMO).

Regarding consent to participate: the IGJ data falls under the Public Access to Government Info Act (Wet openbaarheid van bestuur: WOB). At the beginning of the questionnaires (2014-2017) this obligation was stated to inform the ITCS. For the patients' ratings, the people who rate via the Patients Association (Patientenfederatie) platform agree to their privacy regulations, whereby they agree upon handing over their unanimous data to the Patients Association.

\section{Consent for publication}

\section{Not applicable.}

\section{Competing interests}

The authors declare that they have no competing interests.

\section{Author details}

${ }^{1}$ IQ healthcare, Radboud University and Medical Center, Nijmegen, The Netherlands. ${ }^{2}$ Dutch Health and Youth Care Inspectorate, Utrecht, The Netherlands. ${ }^{3}$ Department of Health Evidence, Radboud University Medical Center, Nijmegen, The Netherlands. ${ }^{4}$ Ministry of Health, Welfare and Sport, The Hague, The Netherlands.

Received: 29 March 2019 Accepted: 27 August 2019

Published online: 21 November 2019

\section{References}

1. Medicare Payment Advisory Commission. Report to the congress: Medicare payment policy in. Washington, DC: MedPAC; 2017.

2. Cylus J, Richardson E, Findley L, Longley M, O'Neill C, Steel D. United Kingdom: Health system review. In: Health Systems in Transition, vol. 17. Copenhagen: World Health Organization 2015 (acting as the host organization for, and secretariat of, the European Observatory on Health Systems and Policies); 2015.
3. Kruse FM. Focusklinieken en betaalbaarheid. In: Jeurissen PPT, Tanke M, Den Haag MH, editors. Betaalbare zorg: Sdu; 2018.

4. Suskind AM, Zhang Y, Dunn RL, Hollingsworth JM, Strope SA, Hollenbeck BK. Understanding the diffusion of ambulatory surgery centers. Surg Innov. 2015;22(3):257-65. https://doi.org/10.1177/1553350614546004.

5. Inspectie Gezondheidszorg en Jeugd: Het resultaat telt Particuliere klinieken. 2016 In. Utrecht: Inspectie Gezondheidszorg en Jeugd. Ministerie van Volksgezondheid, Welzijn en Sport; 2018.

6. Vektis:Aandeel zelfstandige behandelcentra blijft toenemen. Available from: [https://www.vektis.nl/nieuws/aandeel-zelfstandige-behandelcentra-blijfttoenemen]; 2018: Cited 29 Mar 2019.

7. Hollingsworth JM, Saigal CS, Lai JC, Dunn RL, Strope SA, Hollenbeck BK. Surgical quality among Medicare beneficiaries undergoing outpatient urological surgery. J Urol. 2012;188(4):1274-8. https://doi. org/10.1016/j.juro.2012.06.031.

8. Chard J, Kuczawski M, Black N, van der Meulen J, Committee POAS. Outcomes of elective surgery undertaken in independent sector treatment centres and NHS providers in England: audit of patient outcomes in surgery BMJ. 2011;343:d6404. https://doi.org/10.1136/bmj.d6404.

9. Chukmaitov AS, Menachemi N, Brown LS, Saunders C, Brooks RG. A comparative study of quality outcomes in freestanding ambulatory surgery centers and hospital-based outpatient departments: 19972004. Health Serv Res. 2008;43(5 Pt 1):1485-504. https://doi.org/10. 1111/j.1475-6773.2007.00809.x.

10. Trybou J, De Regge M, Gemmel P, Duyck P, Annemans L. Effects of physicianowned specialized facilities in health care: a systematic review. Health Policy. 2014;118(3):316-40. https://doi.org/10.1016/j.healthpol.2014.09.012.

11. Kruse FM, Spierings E, Adang E, Jeurissen P. Marktconcentratie is ook een punt van zorg bij zelfstandige behandelcentra. Economisch Statistische Berichten (ESB). 2018:103(4766):453-5.

12. Luft HS, Bunker JP, Enthoven AC. Should operations be regionalized? The empirical relation between surgical volume and mortality (reprinted from N Engl J med, vol 301, pg 1364-1369, 1979). Clin Orthop Relat R. 2007:457:3-9. https://doi.org/10.1097/BLO.0b013e318034285e.

13. Mesman R, Westert GP, Berden BJMM, Faber MJ. Why do high-volume hospitals achieve better outcomes? A systematic review about intermediate factors in volume-outcome relationships. Health Policy. 2015;119(8):1055-67. https://doi.org/10.1016/j.healthpol.2015.04.005.

14. Dudley RA, Johansen KL, Brand R, Rennie DJ, Milstein A. Selective referral to high-volume hospitals - estimating potentially avoidable deaths. JAMA. 2000;283(9):1159-66. https://doi.org/10.1001/jama.283.9.1159.

15. Halm EA, Lee C, Chassin MR. Is volume related to outcome in health care? A systematic review and methodologic critique of the literature. Ann Intern Med. 2002;137(6):511-20. https://doi.org/10.7326/0003-4819-137-6200209170-00012.

16. Pieper D, Mathes T, Neugebauer E, Eikermann M. State of evidence on the relationship between high-volume hospitals and outcomes in surgery: a systematic review of systematic reviews. J Am Coll Surg. 2013;216(5):101525. https://doi.org/10.1016/j.jamcollsurg.2012.12.049.

17. Chowdhury MM, Dagash H, Pierro A. A systematic review of the impact of volume of surgery and specialization on patient outcome. Br J Surg. 2007; 94(2):145-61. https://doi.org/10.1002/bjs.5714.

18. Varagunam M, Hutchings A, Black N. Relationship between patient-reported outcomes of elective surgery and hospital and consultant volume. Med Care. 2015:53(4):310-6. https://doi.org/10.1097/MLR.0000000000000318.

19. Critchley RJ, Baker PN, Deehan DJ. Does surgical volume affect outcome after primary and revision knee arthroplasty? A systematic review of the literature. Knee. 2012;19(5):513-8. https://doi.org/10.1016/j.knee.2011.11.007.

20. Katz JN, Barrett J, Mahomed NN, Baron JA, Wright J, Losina E. Association between hospital and surgeon procedure volume and the outcomes of total knee replacement. J Bone Joint Surg Am. 2004;86a(9):1909-16. https:// doi.org/10.2106/00004623-200409000-00008

21. Katz JN, Phillips CB, Baron JA, Fossel AH, Mahomed NN, Barrett J, Lingard EA, Harris WH, Poss R, Lew RA, et al. Association of hospital and surgeon volume of total hip replacement with functional status and satisfaction three years following surgery. Arthritis Rheum. 2003;48(2):560-8. https://doi. org/10.1002/art.10754

22. Chattha A, Muste J, Patel A. The impact of hospital volume on clinical and economic outcomes in ventral hernia repair: an analysis with national policy implications. Hernia. 2018;22(5):793-9. https://doi.org/ 10.1007/s10029-018-1803-3. 
23. Chukmaitov A, Devers KJ, Harless DW, Menachemi N, Brooks RG. Strategy, structure, and patient quality outcomes in ambulatory surgery centers (1997-2004). Med Care Res Rev. 2011;68(2):202-25. https://doi.org/10.1177/ 1077558710378523.

24. Chukmaitov AS, Menachemi N, Brown SL, Saunders C, Tang A, Brooks R. Is there a relationship between physician and facility volumes of ambulatory procedures and patient outcomes? J Ambul Care Manage. 2008;31(4):35469. https://doi.org/10.1097/01.JAC.0000336554.46837.a4.

25. Luft HS, Hunt SS, Maerki SC. The volume-outcome relationship practice-makes-perfect or selective-referral patterns. Health Serv Res. 1987;22(2):157-82.

26. Gandjour A, Lauterbach KW. The practice-makes-perfect hypothesis in the context of other production concepts in health care. Am J Med Qual. 2003; 18(4):171-5. https://doi.org/10.1177/106286060301800407.

27. Kraus TW, Buchler MW, Herfarth C. Relationships between volume, efficiency, and quality in surgery - a delicate balance from managerial perspectives. World J Surg. 2005;29(10):1234-40. https://doi.org/10.1007/ s00268-005-7988-5.

28. Gaynor M, Seider H, Vogt WB. The volume-outcome effect, scale economies, and learning-by-doing. Am Econ Rev. 2005;95(2):243-7. https://doi.org/10. 1257/000282805774670329.

29. Christian CK, Gustafson ML, Betensky RA, Daley J, Zinner MJ. The volumeoutcome relationship: Don't believe everything you see. World J Surg. 2005; 29(10):1241-4. https://doi.org/10.1007/s00268-005-7993-8.

30. Hughes RG, Hunt SS, Luft HS. Effects of surgeon volume and hospital volume on quality of Care in Hospitals. Med Care. 1987;25(6):489-503. https://doi.org/10.1097/00005650-198706000-00004.

31. Hollenbeck BK, Daignault S, Dunn RL, Gilbert S, Weizer AZ, Miller DC. Getting under the hood of the volume-outcome relationship for radical cystectomy. J Urol. 2007;177(6):2095-9. https://doi.org/10.1016/ j.juro.2007.01.153.

32. Shortell SM. The evolution of hospital systems: unfulfilled promises and selffulfilling prophesies. Med Care Rev. 1988;45(2):177-214. https://doi.org/10. 1177/107755878804500202

33. Coles JW, Hesterly WS. Transaction costs, quality, and economies of scale: examining contracting choices in the hospital industry. J Corp Finan. 1998; 4(4):321-45. https://doi.org/10.1016/s0929-1199(98)00011-x.

34. Hansmann HB. The role of nonprofit Enterprise. Yale Law J. 1980;89(5):835901. https://doi.org/10.2307/796089.

35. Marsteller JA, Bovbjerg RR, Nichols LM. Nonprofit conversion: theory, evidence, and state policy options. Health Serv Res. 1998;33(5 Pt 2): 1495-535.

36. Newhouse JP. Toward a theory of nonprofit institutions - economic mode of a hospital. Am Econ Rev. 1970;60(1):64-74.

37. Kroneman $M$, Boerma W, van den Berg M, Groenwegen $P$, de Jong J, van Ginneken E. The Netherlands: Health system review. In: Health Systems in Transition, vol. 18. Copenhagen: European Observatory on Health Systems and Policies; 2016.

38. Inspectie Gezondheidszorg en Jeugd (IGJ). Risicoindicatoren. Basisset particuliere klinieken 2015. Utrecht: IGJ; 2015.

39. Kool RB, Kleefstra SM, Borghans I, Atsma F, van de Belt TH. Influence of intensified supervision by health care inspectorates on online patient ratings of hospitals: a multilevel study of more than 43,000 online ratings. J Med Internet Res. 2016;18(7). https://doi.org/10.2196/jmir.5884.

40. Luft HS, Garnick DW, Mark DH, McPhee SJ. Hospital volume, physician volume, and patient outcomes. Assessing the evidence. Ann Arbor: Health Administration Press Perspectives; 1990.

41. Donabedian A. Evaluating the quality of medical care. Milbank Mem Fund Q. 1966:44(3):166-206.

42. Mobin SSN, Keyes GR, Singer R, Yates J, Thompson D. Infections in Outpatient Surgery. Clin Plast Surg. 2013;40(3):439. https://doi.org/10.1016/j. cps.2013.04.009

43. Majholm B, Engbaek J, Bartholdy J, Oerding H, Ahlburg P, Ulrik AMG, Bill L, Langfrits CS, Moller AM. Is day surgery safe? A Danish multicentre study of morbidity after 57,709 day surgery procedures. Acta Anaesthesiol Scand. 2012;56(3):323-31. https://doi.org/10.1111/j.1399-6576.2011.02631.x.

44. Rhee C, Huang SS, Berrios-Torres SI, Kaganov R, Bruce C, Lankiewicz J, Platt R, Yokoe DS. Centers for disease C, prevention prevention epicenters P: surgical site infection surveillance following ambulatory surgery. Infect Control Hosp Epidemiol. 2015;36(2):225-8. https://doi. org/10.1017/ice.2014.23.
45. Reichheld FF. The one number you need to grow. Harv Bus Rev. 2003; 81(12):46.

46. Reid BC, Alberg AJ, Klassen AC, Koch WM, Samet JM. The American Society of Anesthesiologists' class as a comorbidity index in a cohort of head and neck cancer surgical patients. Head Neck. 2001;23(11):985-94. https://doi. org/10.1002/hed.1143.

47. Velleman PF, Hoaglin DC. Applications, basics, and computing of exploratory data analysis Ithaca. N.Y: The Internet-First University Press; 2004

48. Akaike $\mathrm{H}$. Information theory and an extension of the maximum likelihood principle. In: Petrov BN, Csaki F, editors. 2nd International Symposium on Information Theory. Budapest: Akademiai Kiado; 1973. p. 267-81.

49. Mallows CL. Augmented partial residuals. Technometrics. 1986;28(4):313-9 https://doi.org/10.1080/00401706.1986.10488149.

50. Hausman JA. Specification tests in econometrics. Econometrica. 1978;46(6): 1251-71. https://doi.org/10.2307/1913827.

51. Marquardt DW. Generalized inverses, ridge regression, biased linear estimation, and nonlinear estimation. Technometrics. 1970;12(3):591-612. https://doi.org/10.1080/00401706.1970.10488699.

52. Marlow NE, Barraclough B, Collier NA, Dickinson IC, Fawcett J, Graham JC, Maddern GJ. Centralization and the relationship between volume and outcome in knee arthroplasty procedures. ANZ J Surg. 2010;80(4):234-41. https://doi.org/10.1111/j.1445-2197.2010.05243.x.

53. Lin HC, Xirasagar S, Laditka JN. Patient perceptions of service quality in group versus solo practice clinics. Int J Qual Health C. 2004;16(6):437-45. https://doi.org/10.1093/intqhc/mzh072.

54. Ho V, Hamilton BH. Hospital mergers and acquisitions: does market consolidation harm patients? J Health Econ. 2000;19(5):767-91. https://doi. org/10.1016/S0167-6296(00)00052-7.

55. Madison K. Multihospital system membership and patient treatments, expenditures, and outcomes. Health Serv Res. 2004;39(4):749-69. https://doi. org/10.1111/j.1475-6773.2004.00256.x.

56. Cuellar AE, Gertler PJ. How the expansion of hospital systems has affected consumers. Health Aff. 2005;24(1):213-9. https://doi.org/10.1377/hlthaff.24.1.213.

57. Sari N. Do competition and managed care improve quality? Health Econ. 2002;11(7):571-84. https://doi.org/10.1002/hec.726.

58. Kruse FM, Stadhouders NW, Adang EM, Groenewoud S, Jeurissen PP. Do private hospitals outperform public hospitals regarding efficiency, accessibility, and quality of care in the European Union? A literature review. Int J Health Plann Manag. 2018;33(2). https://doi.org/10.1002/hpm.2502.

59. Eggleston K, Shen YC, Lau J, Schmid CH, Chan J. Hospital ownership and quality of care: what explains the different results in the literature? Health Econ. 2008;17(12):1345-62. https://doi.org/10.3386/w12241.

60. Schlesinger M, Gray BH. How nonprofits matter in American medicine, and what to do about it. Health Aff. 2006;25(4):W287-303. https://doi.org/10. 1377/hlthaff.25.w287.

61. Kurlansky PA, Argenziano M, Dunton R, Lancey R, Nast E, Stewart A, Williams T, Zapolanski A, Chang H, Tingley J. Quality, not volume, determines outcome of coronary artery bypass surgery in a university-based community hospital network. J Thorac Cardiovasc Surg. 2012;143(2):287-293.e281. https://doi.org/10.1016/j.jtcvs.2011.10.043.

62. Westbrook JI, Li L, Lehnbom EC, Baysari MT, Braithwaite J, Burke R, Conn C, Day RO. What are incident reports telling us? A comparative study at two Australian hospitals of medication errors identified at audit, detected by staff and reported to an incident system. Int J Qual Health C. 2015;27(1):1-9. https://doi.org/10.1093/intqhc/mzu098.

63. Kruikemeier S, Schäfer W, Coppen R, Friele R. Evaluatie Gefaseerd Toezicht Utrecht: NIVEL; 2010

64. Vermeulen J, Kleefstra S, Zijp E, Kool R. Understanding the impact of supervision on reducing medication risks: an interview study in long-term elderly care. BMC Health Serv Res. 2017;17(1):464. https://doi.org/10.1186/ s12913-017-2418-6.

65. Greaves F, Ramirez-Cano D, Millett C, Darzi A, Donaldson L. Harnessing the cloud of patient experience: using social media to detect poor quality healthcare. BMJ Qual Saf. 2013;22(3):251-5. https://doi.org/10.1136/bmjqs2012-001527.

66. ten Katen M, Eikelenboom S. Winsttrucs zijn overal in de zorg. In: Het Financieele Dagblad; 2017.

\section{Publisher's Note}

Springer Nature remains neutral with regard to jurisdictional claims in published maps and institutional affiliations. 